\title{
Rhomboid beach pattern: A laboratory investigation
}

\author{
O. Devauchelle, ${ }^{1}$ L. Malverti, ${ }^{1}$ É. Lajeunesse, ${ }^{1}$ C. Josserand, ${ }^{2}$ P.-Y. Lagrée, ${ }^{2}$ \\ and F. Métivier ${ }^{1}$ \\ Received 29 July 2009; revised 12 December 2009; accepted 21 January 2010; published 18 June 2010.
}

[1] The formation of beach rhomboid pattern by swash is investigated experimentally. This centimeter-scale structure is classically interpreted as the mark of stationary gravity waves generated by obstacles in supercritical flows. However, thanks to the use of water-based fluids of various viscosity, our experiments show that a rhomboid pattern can develop in subcritical flows. Its angle is primarily a function of the Froude number, as suggested by Woodford (1935), but our data do not support his classical model, nor do they support any of the existing theories. The slowness of the rhombus motion indicates that it is not simply the mark of a hydraulic phenomenon but rather results from the coupling between the water flow and sediment transport.

Citation: Devauchelle, O., L. Malverti, É. Lajeunesse, C. Josserand, P.-Y. Lagrée, and F. Métivier (2010), Rhomboid beach pattern: A laboratory investigation, J. Geophys. Res., 115, F02017, doi:10.1029/2009JF001471.

\section{Introduction}

[2] When observing the surface of a beach, one can very often notice a regular crossed pattern composed of a network of diamond-shaped sand structures (see Figure 1). Their typical size lies from a few centimeters to a few tens of centimeters, while their height rarely exceeds a few millimeters [Stauffer et al., 1976]. They usually appear after the swash, when water returns to sea, hence the name "backwash mark" given by Johnson [1919]. Almost every author refers to this ubiquitous pattern in their own way [Allen, 1982]. In the following, we will use the name rhomboid pattern.

[3] To our knowledge, the first contribution on the subject may be credited to Williamson [1887], whose primary interest was sedimentary records of rhomboid patterns. Indeed, Thompson [1949], Singh [1969] and Collinson and Thompson [1982] reported similar structures in ancient rocks. We are not aware of any other description. Despite this rarity, most studies of present beach rhomboid pattern were motivated by its potential utility as an environmental indicator for ancient shorelines [Hoyt and Henry, 1963].

[4] The majority of publications on the subject refer to small-scale structures (see Otvos [1965] and Stauffer et al. [1976] among others or Allen [1982] for a complete review). However, McMullen and Swift [1967] and Morton [1978] show impressive aerial photographs of at least $10 \mathrm{~m}$ large rhomboid structures, first reported by Straaten [1953]. This suggests that understanding the formation of beach rhomboid pattern could shed light on a more general problem in geomorphology, namely the growth and migration of bed forms, such as ripples, dunes, banks or alternate bars. Rhomboidal

\footnotetext{
${ }^{1}$ Laboratoire de Dynamique des Fluides Géologiques, Institut de Physique du Globe de Paris, Paris, France.

${ }^{2}$ Institut Jean Le Rond d'Alembert, Université Pierre et Marie Curie, CNRS, Paris, France.

Copyright 2010 by the American Geophysical Union. 0148-0227/10/2009JF001471
}

structures resembling the pattern we describe have also been observed in deep (as compared to the bed form size) and turbulent flows [Gyr and Schmid, 1989; Best, 1992; Venditti et al., 2005]. In each of these experiments, the rhomboid shapes appeared on the bed without any other preexisting perturbation and were regular features. The rhombi observed by these authors then transformed into transverse sand waves. We do not know if these structures result from the same mechanism as the experimental pattern described in the present paper.

[5] A number of theories have been proposed to explain the formation of the beach rhomboid pattern (see Table 1). The first quantitative theory is due to Woodford [1935], who noted the likeness between the rhomboid pattern and stationary waves in supercritical open-channel flows. As explained in the caption of Figure 2, the inclination $\alpha$ of the waves with respect to the mean flow direction is easily determined:

$$
\alpha=\arcsin \frac{1}{F},
$$

where $F$ is the Froude number (if $U, D$ and $g$ are the mean velocity of the flow, its mean depth and the acceleration of gravity respectively, then $F=U / \sqrt{g D}$ ). Woodford assumes that the rhomboidal structures the swash carves into the sand are passive marks of these waves, and thus should present the same angle.

[6] Chang and Simons [1970] were the first to write a full system of equations which includes both the water flow and the sediment transport. By doing so, they introduced the idea that rhomboid patterns could result from the coupled interaction between water flow and sediment transport. However, even though Chang and Simons [1970] have written a fully coupled system of equations, they later neglect sediment transport, thus returning to Woodford's model. They would have ended up with Woodford's formula but for a mistake in their analysis (see Appendix A). 


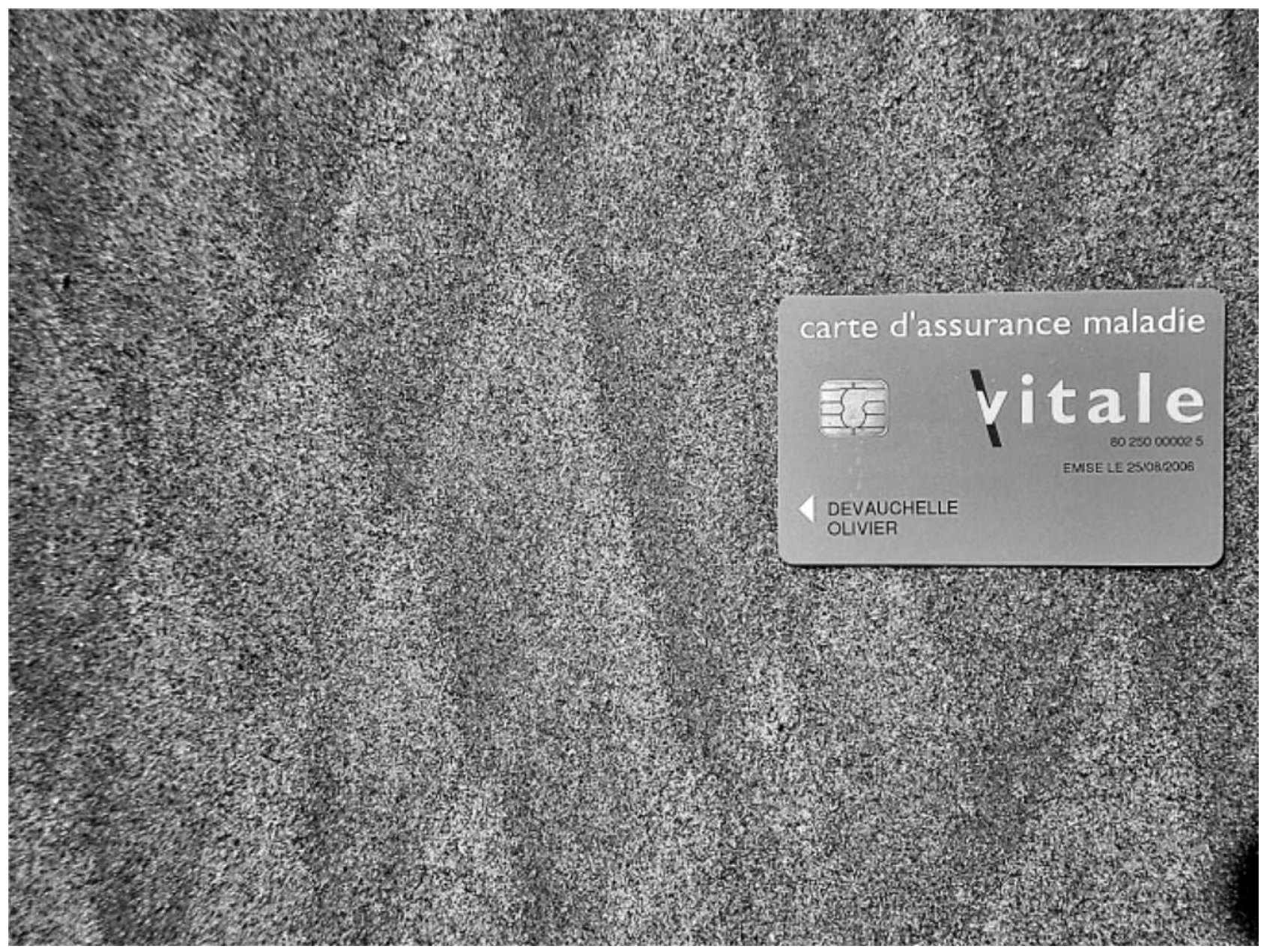

Figure 1. Rhomboid beach pattern on the beach at Goleta, California. Such features form on the swash zone, when a thin film of water returns to sea. Here the structure is visualized by the segregation between grains of different colors. The short dimension of the card is $5.4 \mathrm{~cm}$.

[7] The question of the perturbation from which a rhomboid pattern originates was raised early in the history of analysis of the pattern. Otvos suggested that the elements in rhomboids were associated with triggering obstacles on the bed ("shell fragments, pebbles, plant-clumps, et cetera" [Otvos, 1965, p. 271]). However, on natural surfaces these obstacles will be arranged at random, in contradiction with a striking feature of rhomboid pattern, namely its regular wavelength (see Figure 1). This was already pointed out by Woodford, who warned the reader about the "V-shaped grooves which spread from the snouts of partly buried sand crabs" [Woodford, 1935, p. 518]. Karcz and Kersey [1980] performed experiments on well-sorted sand, and were able to generate regular rhomboid pattern, thus showing that this pattern can appear spontaneously without any obstacle. Later, Daerr et al. [2003] reported the same pattern on a plate covered with a uniform sediment, when it is withdrawn at constant angle and velocity from a bath of still water, again without any obstacle.

[8] In Woodford's theory, the rhomboid pattern has to be generated by some obstacle. Allen's remark "It appears that symmetrically interfering oblique hydraulic jumps can also be produced in channelized flows of sufficient breadth without deflecting obstacle" [Allen, 1982, p. 399] is probably

Table 1. Different Theories for the Formation of the Rhomboid Pattern, by Order of Publication ${ }^{\mathrm{a}}$

\begin{tabular}{|c|c|c|}
\hline Mechanism & Angle Expression & References \\
\hline Two sets of superimposed ordinary ripples & & Williamson [1887] \\
\hline Stationary wave in a supercritical flow (see Figure 2) & $\alpha=\arcsin (1 / F)$ & Woodford [1935] \\
\hline Drainage trough deposited sand (no surface flow required) & & Stauffer et al. [1976] \\
\hline Hydraulic jumps & $\begin{array}{c}\alpha=\arctan (1 / F) \text { corrected to } \alpha=\arcsin (1 / F) \\
\text { in Appendix A }\end{array}$ & Chang and Simons [1970]; Allen [1982] \\
\hline Interaction between antidunes and longitudinal striations & $\alpha=\arctan \left(1 /\left(\pi F^{2}\right)\right)$ (in turbulent flows) & Ikeda $[1983]$ \\
\hline Moving contact line instability & & Devauchelle et al. [2007] \\
\hline
\end{tabular}

\footnotetext{
${ }^{a}$ An extensive review was presented by Allen [1982].
} 


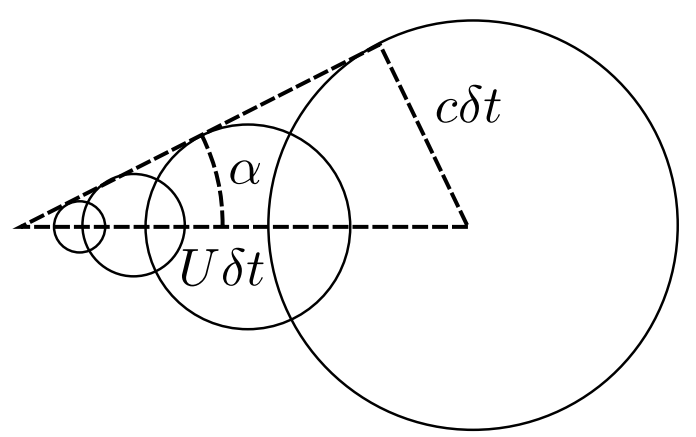

Figure 2. Scheme of the gravity wave propagation in a supercritical flow. A perturbation propagates in all directions at velocity $c$, while it is transported by the mean flow at velocity $U$. For supercritical flows (that is, for $c>U$ ), the angle $\alpha$ of the wave envelope satisfies the relation $\sin (\alpha)=c / U=1 / F$, where $F$ is the Froude number. Woodford [1935] first suggested that such stationary waves could produce the rhomboid pattern.

based on the experiments described by Chang and Simons [1970], during which regularly spaced fronts were formed. However, we believe this remark is somewhat misleading, since the "hydraulic jumps" of the experiment were formed in interaction with the granular bed. Stationary hydraulic jumps, without this interaction, do not present any characteristic wavelength and must be triggered by obstacles. To our knowledge, regular rhombi such as the ones of Chang and
Simons [1970] have been observed only in situations where the flow interacts with its granular substrate, as in the experiments presented here.

[9] More recently, Stauffer et al. [1976] proposed a radically different theory based on the underground flow induced by the beach drainage. It has not lead to quantitative predictions of the rhomboids characteristics, and thus cannot be tested against experiments or field measurements. Later, Devauchelle et al. [2007] showed that the moving contact line (the intersection of the water surface with the sediment) is not responsible for the rhomboid pattern, at least in the experiments of Daerr et al. [2003].

[10] In order to test the above theories, we have performed a series of laboratory experiments in a laminar channel. The measurements focus on the geometrical properties of the pattern, namely its angle $\alpha$ and its wavelength $\lambda$, which are critical for quantitative comparison against theory. The velocity of the experimental bed forms then provides some insight about the pattern dynamics. Preliminary experiments in a smaller channel were described by Devauchelle et al. [2008].

\section{Description of the Experiment}

\subsection{Experimental Arrangements and Procedure}

[11] We performed this set of experiments in a flume of width $W=9.6 \mathrm{~cm}$ and length $L=240 \mathrm{~cm}$ (Figure 3). The flume reposed on a tilted plane which allowed us to vary the mean bed slope $S$, measured with a digital inclinometer of
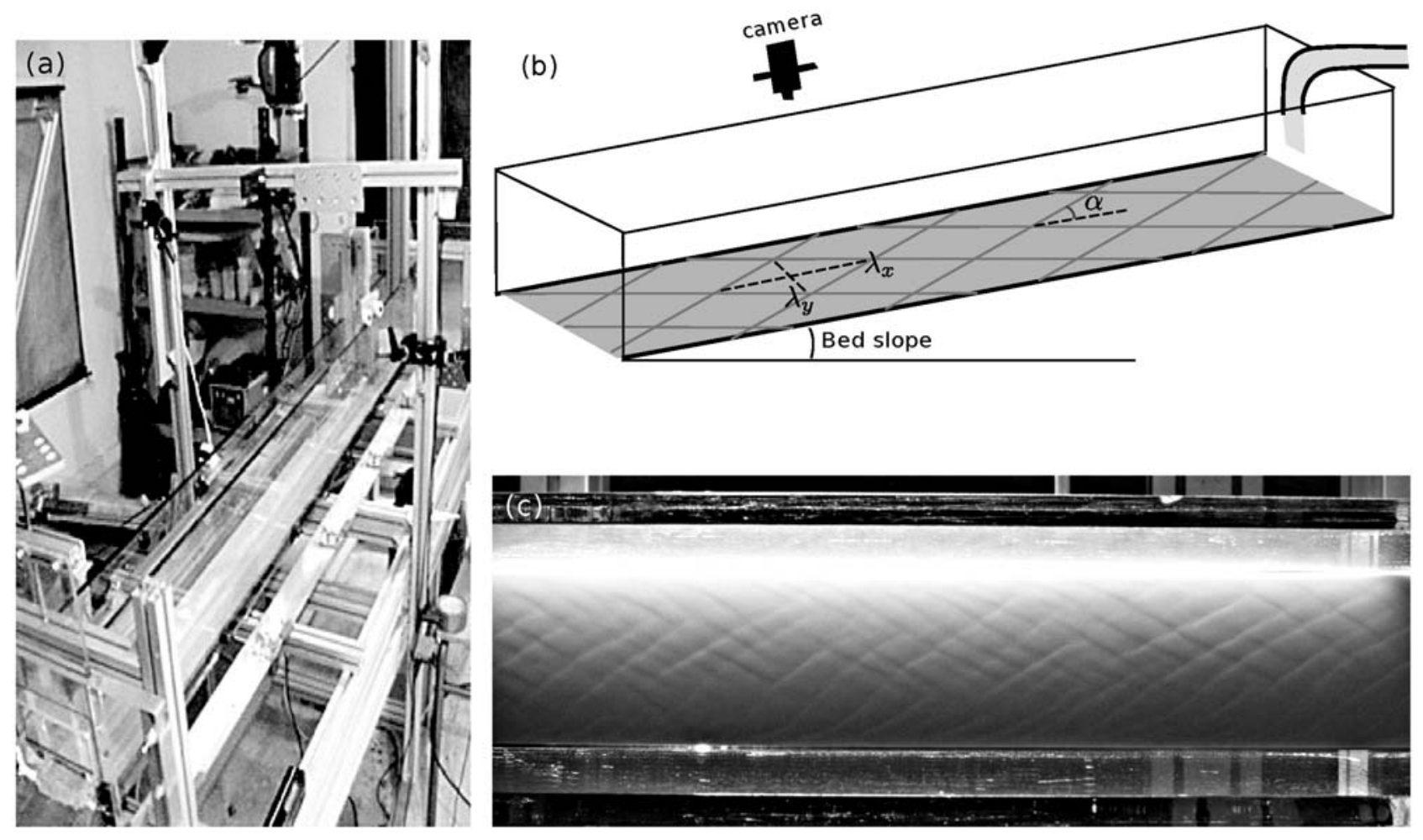

Figure 3. (a) Picture of the experimental arrangement. The glass channel is filled with silica grains, above which a thin film of water flows. Pictures of the bed forms are taken from above. (b) Schematic of the experimental setup, showing the definition of the rhomboid pattern angle and wavelengths. (c) Example of observed rhomboidal bed forms (flow is from right to left). 


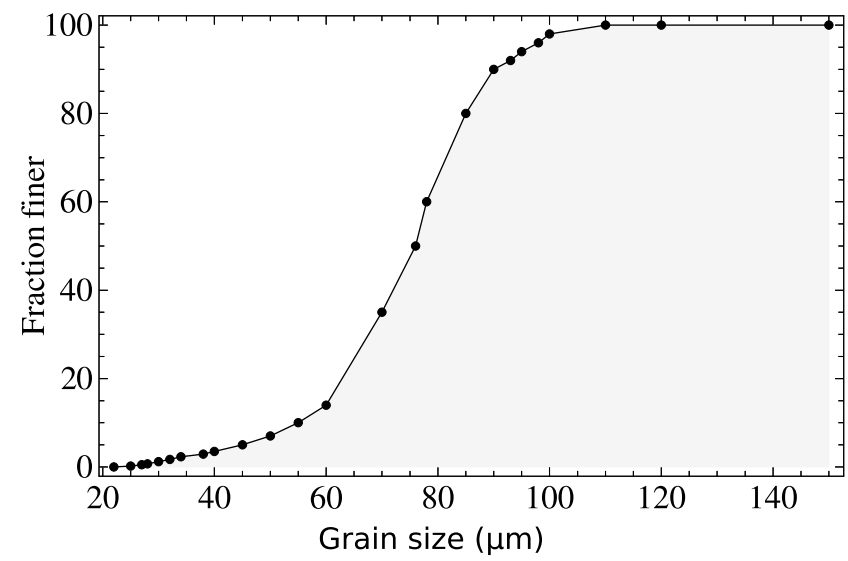

Figure 4. Cumulative distribution function of the sediment used in the experiment. The median grain diameter $d_{s}$ is about $75 \mu \mathrm{m}$.

accuracy $0.1^{\circ}$. The flume was filled with a $5.5 \mathrm{~cm}$ thick bed of glass beads of density $\rho_{s}=2500 \mathrm{~kg} \mathrm{~m}^{-3}$. The sediment grain diameter distribution (Figure 4) had a geometric standard deviation of $\sigma_{g}=\sqrt{d_{84} / d_{16}}=1.2$, where $84 \%$ (respectively $16 \%$ ) of the grains have a diameter below $d_{84}$ (respectively $d_{16}$ ) [Vanoni, 2006]. In the following, we will consider this well-sorted sediment as monodisperse, with a median grain diameter $d_{s} \approx 75 \mu \mathrm{m}$.

[12] A pump injected the fluid at the flume inlet. The flow discharge $Q_{w}$ remained constant during each experimental run, and was measured with a flowmeter of accuracy $0.01 \ell \mathrm{min}^{-1}$. The fluid was either pure water or a mixture of water and glucose, which mass proportion varied from $0 \%$ to $50 \%$. The slope ranged from 0.008 to 0.052 (that is from $0.46^{\circ}$ to $3^{\circ}$ ), the water discharge from $0.24 \ell \mathrm{min}^{-1}$ to $4.42 \ell \mathrm{min}^{-1}$ and the fluid viscosity from $10^{-6}$ to $5.6 \times 10^{-6} \mathrm{~m}^{2} \mathrm{~s}^{-1}$.

[13] We also measured the sediment discharge $Q_{s}$, by continuously weighing a tank fitted with an overflow, into which were collected both the sediment and the water leaving the channel. However, this measurement was only possible when the sediment discharge was large enough to be measured during the time of an experiment.

[14] The influence of glucose on surface tension is fairly moderate. For instance, adding $17 \%$ of glucose to water produces a surface tension increase of less than $2 \%$, while adding $55 \%$ of glucose creates an increase of about $5 \%$ [Docoslis et al., 2000]. We will thus approximate the surface tension of the mixture by the pure water value, $\sigma=74 \times$ $10^{-3} \mathrm{Nm}^{-1}$. It was not measured during the experiments.

[15] The Reynolds number is defined as

$$
\operatorname{Re}=\frac{U D}{\nu}=\frac{Q_{w}}{W \nu}
$$

where $D$ and $U$ are the flow depth and the average water velocity respectively. The Reynolds number below which an open channel flow may be considered laminar is typically 500 [Orszag and Kells, 1980]. Since it varied between 9.9 and 420 during our experiments, we will hereafter assume that the flow is laminar.

[16] The flow depth was too small to be measured with sufficient precision. Instead, it can be approximated by the depth of a Nusselt film with a parabolic velocity profile, as confirmed by Malverti et al. [2008] with the same experimental setup. The mass and momentum balance for a Nusselt film reads

$$
Q_{w}=W D U, \quad g S=\frac{3 \nu U}{D^{2}},
$$

where $g$ is the acceleration of gravity. Based on the above equations, the water depth ranged between $0.89 \mathrm{~mm}$ and $5.5 \mathrm{~mm}$ during our experiments, while the averaged velocity varied between $0.30 \mathrm{~ms}^{-1}$ and $0.037 \mathrm{~ms}^{-1}$.

[17] The experimental procedure was the following. An initially flat bed was prepared by sweeping a rake over the sediment surface, the tilt and height of the rake being constrained by two rails parallel to the channel. This flat bed is referred to as the base state in the following. Under certain conditions, a rhomboidal bed form appeared at the sediment surface a few seconds after the flow had started (see section 3.1). The rhomboid pattern was extremely flat (typically less than $1 \mathrm{~mm}$ ), and usually disappeared (or at least became even fainter) when the water flow stopped. In order to make these faint bed forms visible, the bed was lit up with a light beam directed horizontally through one of the sides. We then recorded frames of these patterns at regular time intervals with a camera fixed above the flume (Figure 3 ). We measured the opening angle $\alpha$, the longitudinal and transverse wave length ( $\lambda_{x}$ and $\lambda_{y}$ respectively) and the velocity of the bed forms. The angle $\alpha$ corresponds to the angle between the crest line of a bed form and the direction of the flow (see Figure 3 ). The longitudinal wavelength $\lambda_{x}$ was estimated by counting the number of structures along the flume bed in the field of view. The same method was used to estimate $\lambda_{y}$. The pattern wavelength $\lambda$, which represents the distance between two opposite sides of a rhombus, is given by

$$
\lambda=\frac{1}{\sqrt{1 / \lambda_{x}^{2}+1 / \lambda_{y}^{2}}} .
$$

\subsection{Dimensional Analysis}

[18] Ten experimental parameters are likely to influence the rhomboid pattern: (1) the water discharge $Q_{w}$; (2) the slope $S$; (3) the fluid viscosity $\nu$; (4) the acceleration of gravity $g$; (5) the fluid surface tension $\sigma ;(6)$ the density of water $\rho_{w}$ and that of the grains $\rho_{s} ;(7)$ the median grain size $d_{s}$; and (8) the width and the length of the channel, $W$ and $L$, respectively.

[19] The above list includes only the parameters on which we have a direct control. Of course, flow parameters such as the velocity $U$ or the depth $D$ are likely to influence the pattern shape. Nevertheless, they are selected by the arrangement itself once the operator sets the discharge, the slope and the viscosity. Consequently, in the dimensional analysis, they are experimental results to be considered in the same way as the bed forms characteristics. Below, the influence of the flow depth and velocity on the bed pattern is expressed through dimensionless numbers which can be evaluated form the primary quantities listed above.

[20] According to the so-called Pi theorem [Barenblatt, 1996], and since there are three dimensions of interest (namely length, time and mass), the results are functions of 

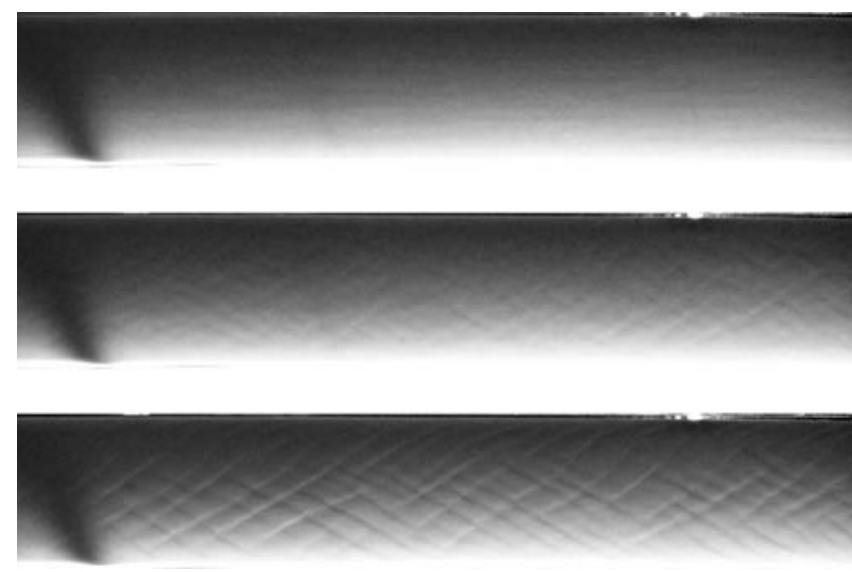

Figure 5. Development of a rhomboid instability on the granular bed of a laminar channel (flow is from right to left). From top to bottom, the pictures were taken at times $t=0 \mathrm{~s}, t=$ $90 \mathrm{~s}$, and $t=282 \mathrm{~s}$, respectively. The pattern develops roughly uniformly in terms of amplitude, angle, and wavelength. Its formation does not involve ripples. For this run, $F=1.1$, $S=0.022$, Bo $=0.58$, and $\theta=0.40$.

any set of seven independent nondimensional numbers. Given that only three dimensional parameters varied during the experiment $\left(Q_{w}, S\right.$ and $\nu$ ), it is natural to define a set of three varying nondimensional numbers, and four nondimensional constants. We first define the following constants (the symbol $\equiv$ denotes a definition): (1) the density ratio $R \equiv$ $\left(\rho_{s}-\rho_{w}\right) / \rho_{w} \approx 1.50 ;(2)$ the channel aspect ratio $R_{c} \equiv W / L \approx$ 0.040 ; (3) the nondimensional grain size $R_{s} \equiv d_{s} / W \approx 7.81 \times$ $10^{-4}$; and (4) the Bond number Bo $\equiv \rho g W^{2} / \sigma \approx 1240$.

[21] The Bond number is the squared ratio of the channel width to the capillary length. The experimental results are likely to depend on the above constants, but our experiments can provide no information about their influence. Finally, we choose three varying independent numbers: (1) the channel slope $S ;(2)$ the Froude number $F \equiv\left(S Q_{w} /(3 W \nu)\right)^{1 / 2}=$ $U / \sqrt{g D}$; and (3) the Shields parameter $\theta \equiv\left(3 \nu Q_{w} S^{2} /\right.$ $\left.\left(g W R^{3} d_{s}^{3}\right)\right)^{1 / 3}=\tau /\left(\left(\rho_{s}-\rho\right) g d_{s}\right)$, where $\tau$ is the shear stress exerted by the flow on the bed.

[22] The above definitions involve only experimental parameters which we can measure directly. Of course, they correspond to the classical definitions through equation (3). The Froude number $F$ relates inertial forces to pressure, when the pressure field is hydrostatic. It is also the ratio of celerity of the surface gravity wave to flow velocity, to the extent that the shallow water approximation holds. The Shields parameter is the ratio of the viscous force exerted by the flow on a bed particle to its immersed weight. There exists a threshold value of the Shields number $\theta_{c}$ below which no sediment is transported. During our experiments, the flow was slow enough and the particles large enough for the grains to remain near the bed during transport. As a consequence, the dominant transport mode is bed load [Malverti et al., 2008], which is usually characterized by the Shields parameter [Vanoni, 2006].

[23] The choice of the above parameters is arbitrary, any set of three independent and varying quantities would be sufficient to describe the results. However, we define three additional quantities for illustrative purpose. They can be derived from the initial set of parameters: (1) the Reynolds number defined in section 2.1, which satisfies $\mathrm{Re}=3 F^{2} / S$; (20 the Weber number We $\equiv\left(\theta R R_{S} F\right)^{2} \mathrm{Bo} / S=\rho U^{2} D / \sigma$; and (3) the cross-section aspect ratio of the flow $R_{f} \equiv \sqrt{\mathrm{We} / \mathrm{Bo}} / F=D / W$.

[24] The Reynolds number compares viscous forces to inertia, while the Weber number compares the influence of capillary forces to fluid inertia.

\section{Results}

\subsection{Description of the Bed Forms}

[25] When the water pump is turned on, the flow fills the channel, without inducing any visible deformation of the initial bed. For a very small discharge, no sediment grain moves. Above a certain discharge, a few grains move at the sediment surface, indicating that the Shields parameter is above the threshold. The system may then evolve in three different ways.

[26] In some cases (typically for high slope $S$ and high outflow $Q_{w}$ ), the bed remains flat and homogeneous, despite a visible sediment flux along the flow direction $x$. The system is then stable.

[27] In many cases, a rhomboid pattern slowly grows on the sediment surface, as illustrated by Figure 5 . The time needed to reach a quasi-static regime is typically a few seconds. The formation of the rhomboid pattern does not involve any visible intermediate structure. On the larger part of the field of view, the pattern is regular enough to measure its wavelength and an inclination with respect to the mean flow. As already noted by Karcz and Kersey [1980], the sand waves are not generated by any obvious initial perturbation, although they can be artificially generated by digging a small hole in the sand (or by making a small sand bump). Every pattern we observed migrated slowly downward (typically at 0.5 to $1 \mathrm{~mm} \mathrm{~s}^{-1}$ ), a characteristic also observed in the field [Hoyt and Henry, 1963]. Once the equilibrium shape is reached, the rhomboid pattern can be described as the crisscrossing of inclined straight lines, each line corresponding to a sharp front in the sediment-surface elevation. This front is the lee side of a sandbank, while the stoss side is gently sloping between two fronts (see Figure 6). The height of this bed form scarcely ever exceeds a millimeter, and remains generally small as compared to flow depth. Figure 7 shows examples of the various wavelengths and angles we observed for the rhomboid pattern. The angle varied between $10^{\circ}$ and $90^{\circ}$. The wavelength was usually a few centimeters, or a few tens of centimeters.

[28] The last type of bed form that formed during our experiment were sand waves perpendicular to the main flow (at least initially), which will be named ripple hereafter, even though there is no universal definition for this term [Coleman and Eling, 2000; Charru and Mouilleron-Arnould, 2002]. This pattern remains roughly perpendicular to the flow, although secondary instabilities may deform its initially regular shape. At the highest Reynolds numbers, such deformations of the ripples could lead to triangular structures. However, both their shape and their obvious relation with initially transverse sand waves allowed us to discriminate between them and true rhomboid patterns. The ripple wavelength in our experiment was typically a few centimeters. They usually form alone, but we have observed them in association with a rhomboid pattern, as shown on Figure 7c. 

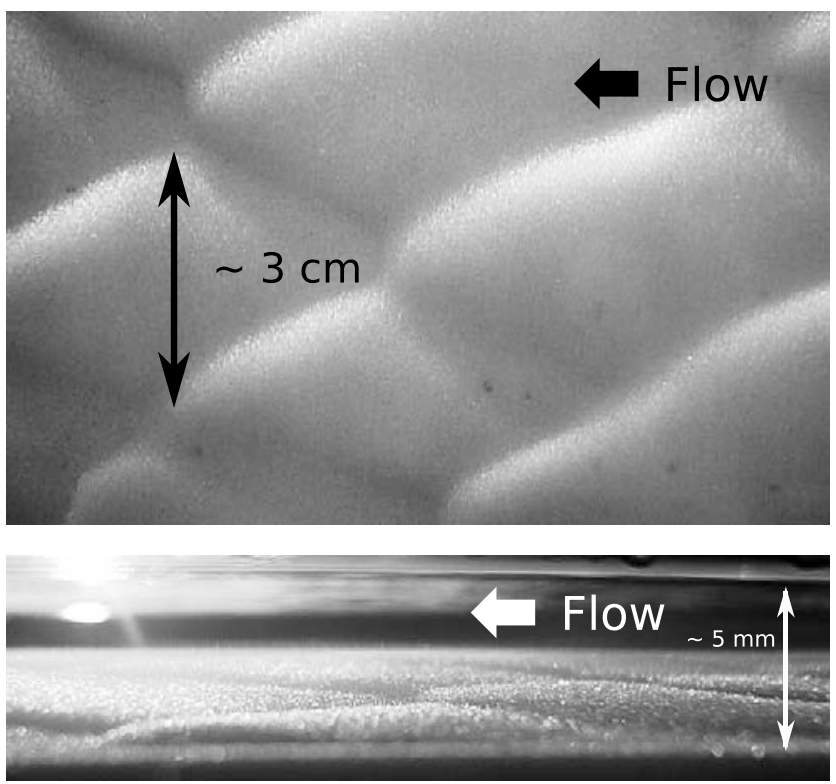

Figure 6. Close view of an experimental rhomboid pattern. (top) Rhomboidal pattern observed through the water surface. For this run, $F=0.43, S=0.013, \mathrm{Bo}=1.35$, and $\theta=0.37$. (bottom) Rhomboidal pattern observed through the glass sidewall of the experimental channel. The amplitude of the rhomboid pattern is of order, or less than, $1 \mathrm{~mm}$.

Ripples and rhomboid pattern thus behave as independent structures. However, when the angle of the rhomboid pattern tends to $90^{\circ}$, it becomes impossible to discriminate between the two types of bed forms.

\subsection{Influence of the Experimental Parameters}

[29] The shape of the rhomboid pattern changes as the slope, the discharge and the viscosity are varied. Figure 8 expresses this dependence with respect to six nondimen- sional parameters. However, our exploratory experiments allowed us to vary only three parameters independently. Definitive experiments should isolate the parameter variations, for instance by means of different grains size and adjustable channel width. For that reason, and in order to identify the most significant correlations, we have computed Spearman's rank correlation coefficient for each graph (the rank correlation coefficient is more relevant than the classical correlation coefficient when one do not expect any specific relation [Spearman, 1904]). The product of the correlation coefficient for the angle by the correlation coefficient for the wavelength then quantifies the influence of a parameter on the pattern shape (Table 2). In Figure 8, the plots are ordered from strong to weak correlation (this order remains unchanged if the classical correlation coefficient is used instead of the rank correlation).

[30] The parameter influencing most the pattern shape is the Froude number, as suggested initially by Woodford [1935]. The pattern angle decreases from about $85^{\circ}$ to about $10^{\circ}$ as the Froude number increases from 0.5 to 2.5 . Varying the Froude number thus induces a shift from almost transverse bed forms to elongated rhombus. Increasing the Froude number also induces an increase in the pattern (nondimensional) wavelength, from about 2 to a few tens.

[31] The influence of the channel slope on the pattern shape is similar to that of the Froude number, although the correlation is weaker. This is most likely due to the nature of our arrangements, which impose a relation between slope and Froude number. The slope-Froude number effects are sorted out in Figure 10.

[32] As the Shields parameter decreases from about 0.7 to about 0.45 , the pattern angle increases. From 0.45 to 0.2 , the data are separated into two sets, one with a roughly constant angle of about $25^{\circ}$, the other showing a increase form $40^{\circ}$ to $85^{\circ}$ as the Shields parameter decreases. As this parameter increases, the nondimensional wavelength also increases, although the scatter is wide. The lowest value of the Shields parameter on these plots (about 0.22 ) does not correspond
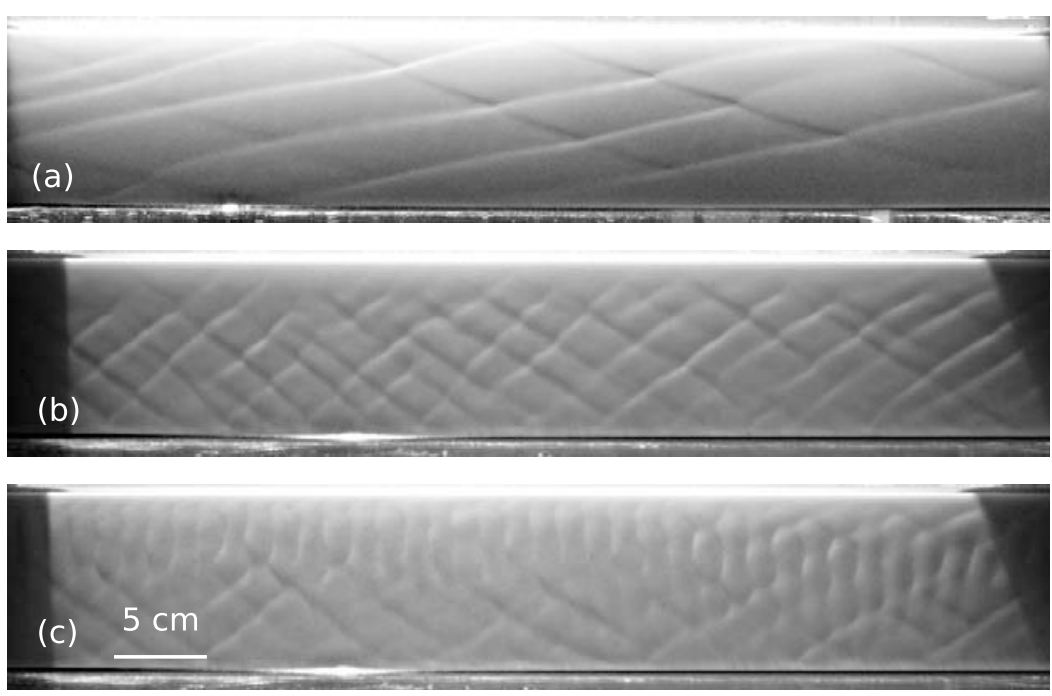

Figure 7. Various bed forms observed in our experiment (flow is from right to left). (a) Large rhomboid pattern $(F=1.76, S=0.03$, and $\theta=0.616)$. (b) Small rhomboid pattern $(F=0.95, S=0.015$, and $\theta=0.485)$. (c) Rhomboid pattern mixed with ripples $(F=1.01, S=0.015$, and $\theta=0.504)$. 

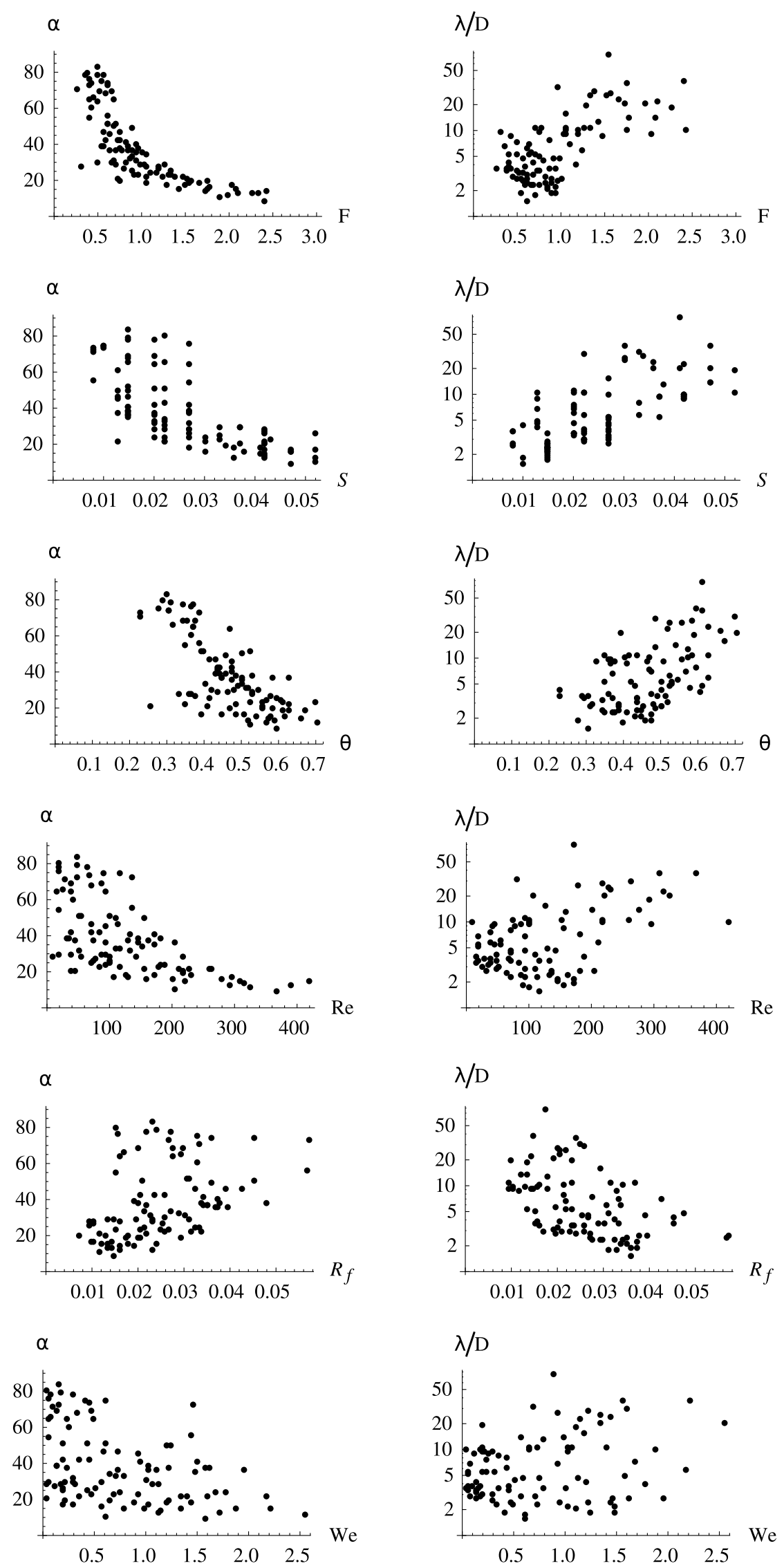

Figure 8. Geometrical properties of the rhomboid pattern (angle $\alpha$ and nondimensional wavelength $\lambda / D$ ) versus various nondimensional parameters. The complete set of experiments is represented here. 
Table 2. Correlation Matrix for the Dependence of the Pattern Angle and Wavelength on Six Nondimensional Parameters ${ }^{\mathrm{a}}$

\begin{tabular}{ccccccc}
\hline Pattern Property & $F$ & $S$ & $\theta$ & $\mathrm{Re}$ & $R_{f}$ & $\mathrm{We}$ \\
\hline$\alpha$ & -0.878 & -0.755 & -0.729 & -0.663 & 0.441 & -0.472 \\
$\lambda D$ & 0.583 & 0.685 & 0.512 & 0.348 & -0.521 & 0.165
\end{tabular}

${ }^{\mathrm{a}}$ The coefficients correspond to Spearman's rank correlation.

to a global threshold for sediment transport, but rather to the absence of any bed instability.

[33] At low values of the Reynolds number (below 100), the pattern angle can be anything between $15^{\circ}$ and $85^{\circ}$. However, its range shrinks as the Reynolds number increases, to the point that only elongated patterns are observed at Reynolds numbers larger than about 300. The Reynolds number also presents a weak positive correlation with the pattern wavelength.

[34] The flow aspect ratio is poorly correlated with the pattern shape. Deeper flows tend to form shorter and less elongated rhombus.

[35] Finally, the influence of the Weber number on the pattern is very weak. At higher Weber numbers, the pattern seems to be more elongated, and to present a slightly larger wavelength. One cannot conclude from the weakness of this correlation that capillary forces have no effect on the bed forms, only that our experiments were not designed to evaluate this influence.

[36] Since only three parameters were varied during the experiment, we will hereafter present our results in terms of the three parameters which have the strongest influence on the pattern, namely $F, S$ and $\theta$.

[37] Figure 9 illustrates in more detail the dependence of the pattern angle with respect to the Froude number. Even though Woodford's theory reproduces qualitatively the dependence of the angle with respect to the Froude number, the curve corresponding to relation (1) unquestionably lies outside the experimental error bars. However, an empirical relation inspired from equation (1), namely

$$
\alpha \approx \arcsin \frac{0.463}{F},
$$

provides a reasonably good fit to the data. This empirical curve tends to $\alpha=90^{\circ}$ (that is, a ripple-like pattern) around $F=0.463$. Figure 9 clearly shows that a rhomboid pattern can develop in a subcritical flow (that is, for a Froude number below one), in contradiction with Woodford's theory. Our experiments thus show that the rhomboid pattern does not result from stationary waves forming in supercritical flows. One could include capillary forces into Woodford's theory, by replacing the Froude number in relation (1) with $U / c$, where $c$ is the celerity of gravity-capillary waves in shallow water. However, since capillarity makes the surface waves faster, this would lead to predictions further away from the data.

[38] The diagram of Figure 10 provides an explanation for the apparent contradiction between our results and those of Karcz and Kersey [1980]. Indeed, these authors observed no rhomboid pattern below the curve $S=3 / \mathrm{Re}$, which corresponds to the vertical line $F=1$ in the $(F, S)$ plane. On the contrary, we obtained many rhombi in subcritical flows when the water was mixed with glucose. Except for one exception, we did not observe any subcritical rhombus without glucose. This result indicates that subcritical rhombi appear extremely seldom if the water viscosity is not increased. Therefore Karcz and Kersey, using only unmixed water, were unlikely to observe any subcritical rhombus.

[39] Even though the pattern shape depends mostly on the Froude number, the role of the channel slope cannot be

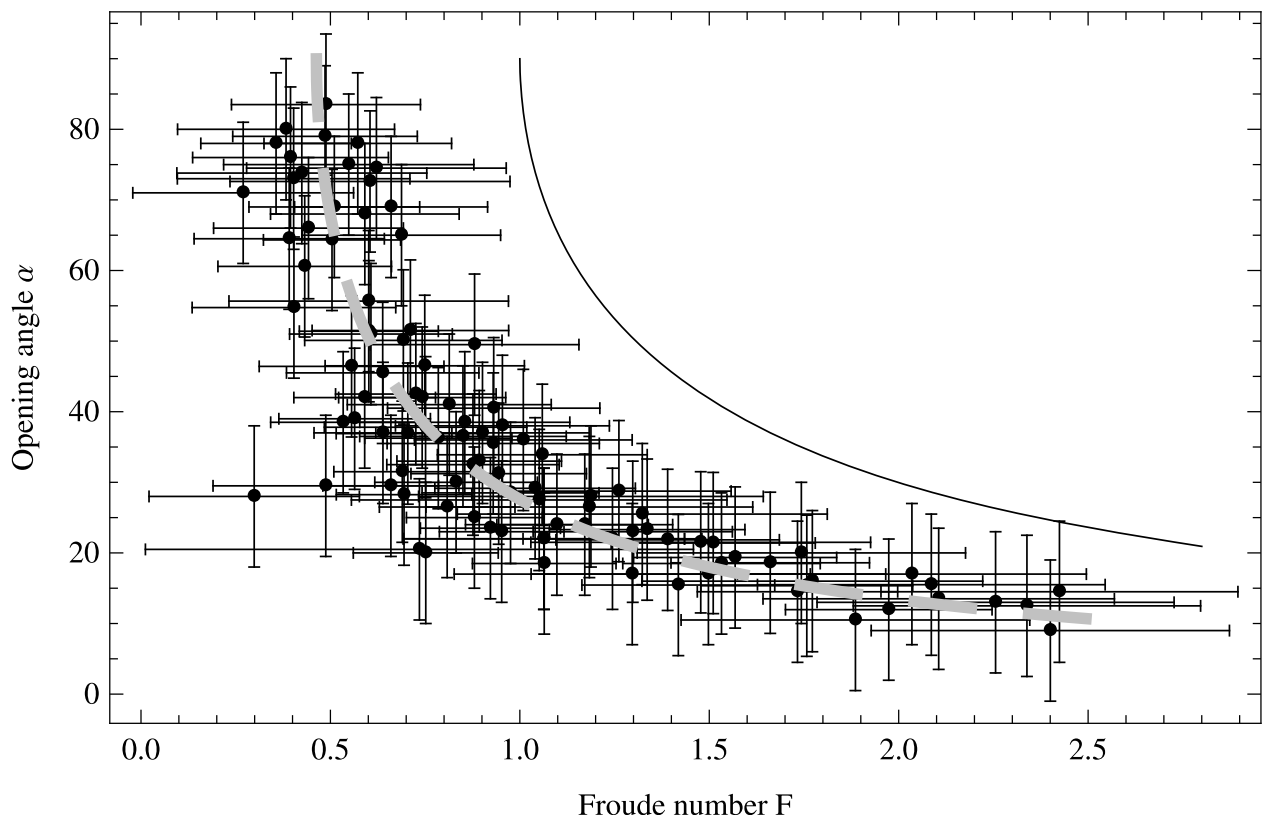

Figure 9. Rhomboid pattern angle as a function of the Froude number. Complete set of experimental results with error bars. Black solid line, relation (1), corresponding to the theory of Woodford [1935]. Thick gray dashed line, best fit corresponding to relation (5). 


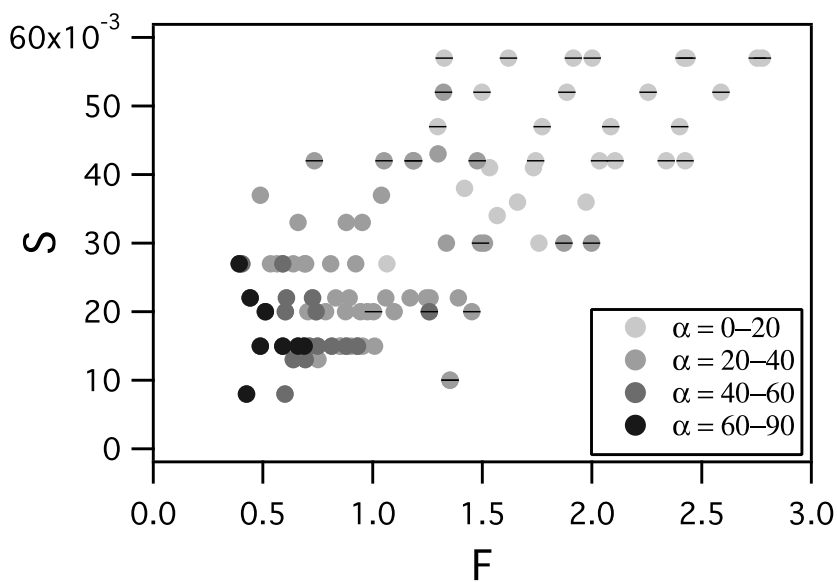

Figure 10. Existence diagram of the rhomboid instability versus the Froude number $F$ and the channel slope $S$. The gray scale indicates the angle of the corresponding pattern and thus may be used to discriminate between ripples and rhomboid structures. The dashed disks represent data obtained with pure water, whereas the fluid viscosity was increased with sugar otherwise.

neglected, as illustrated in Figure 11. If the Froude number varies while the slope is fixed to 0.015 (circles), the relation between the pattern angle and the Froude number shows less dispersion than in Figure 8. If the slope is fixed to 0.03 (squares), the correlation remains, but the whole curve is shifted downward with respect to the previous case. Finally, when the slope is fixed to 0.05 (disks), the data still line up onto the continuation of the previous relation. Thus, the pattern angle is not a function of the Froude number only, and part of the dispersion observed in Figure 11 can be ascribed to variations in the channel slope.

[40] Hoyt and Henry [1963] related the rhombus aspect ratio (that is, the angle $\alpha$ ) to the slope of the beach where they formed. Their data set is rather convincing (see their Figure 3), but they did not measure the Froude number, which is likely to change both with the location of the measurements, and during the pattern formation. The affine relation between $\alpha$ and $S$ they propose probably results from a correlation between the slope and the other flow parameters. It might still be used as an indicator of the paleoenvironment, but the data on which it is based are not sufficiently controlled for their conclusion to hold in the general case.

\subsection{Dynamics of the Bed Forms}

[41] Our experiments highlight the limitations of theories based on supercritical surface waves. We suspect that their main flaw is to consider only the fluid flow, without coupling it to sediment transport. The present section is devoted to the evaluation of this proposition. Indeed, bed load transport is slow as compared to the water velocity. Consequently, if the sediment transport is strongly coupled to the flow, the typical time scale of the bed forms dynamics should be controlled by bed load transport.

[42] Rhomboid patterns typically have a steep lee side, and a gently sloping stoss side (Figure 6). Let us denote by $h_{+}$and $h_{-}$the elevation of the rhombus crest, and the elevation of its bottom (Figure 12). Similarly, $q_{s^{+}}$and $q_{s^{-}}$denote respectively the sediment flux at the crest and at the bottom (the sand compaction is included in this definition). Now, if the pattern has reached a steady state and moves at velocity $c_{r}$, the sediment mass balance reads

$$
c_{r}\left(h_{+}-h_{-}\right)=q_{s+}-q_{s-} .
$$

The above equation is the integral of the Exner equation.

[43] At the lowermost point of the pattern, the grains are sheltered from the flow, and one can expect the bed load to be very low there. Although we have no strict evidence for such a statement, the grains indeed seemed immobile to the eye near the bottom of the pattern during the experiments. We will assume hereafter that $q_{s^{-}} \approx 0$ as a first approximation.

[44] Now, the average sediment discharge $Q_{s}$ measured during some of the experiments provides a rough approximation for the bed load transport upstream of the crest, that is $q_{s^{+}} \approx Q_{s} / W$. Thus, assuming further that the front amplitude $\delta h=h_{+}-h_{-}$does not vary much between experimental runs, the pattern velocity $c_{r}$ should be strongly related to the average sediment discharge.

[45] One can extract the pattern velocity from the photographs of the bed taken at regular time intervals. This procedure requires that the pattern can be recognized unambiguously on a sufficient number of successive pictures. This constraint, in addition to the difficulty in measuring the sediment discharge (see section 2.1 ) reduces greatly the quantity of data.

[46] Figure 13 shows the pattern velocity as a function of the sediment discharge, when both quantities can be measured. Despite the small number of data points, the two quantities appear to be strongly correlated (the correlation coefficient is 0.85 ). The best linear fit gives an average value of $\delta h=0.49 \mathrm{~mm}$ for the front amplitude, which is correct to well within an order of magnitude. This result is clearly preliminary, since it concerns a quantity that is not easy to measure precisely, and which can be predicted by no theory. However, it supports the idea that the time scale involved in the rhomboid pattern dynamics is that of bed load transport.

[47] If a quantitative model of the rhomboid pattern can be elaborated, the tight relation between the rhomboid pattern and sediment transport could be used to test transport models, as ripples have been used [see, e.g., Charru, 2006]. The rhomboid pattern provides one more measurable quantity than the ripples, namely their opening angle. This point could prove essential when tackling the difficult issue of the lateral slope effect on sediment transport.

\section{Discussion and Conclusion}

[48] The present paper reports experimental investigations of the rhomboid pattern. It is demonstrated that bed forms similar to the rhombus commonly found on beaches can develop spontaneously on a granular bed activated by a thin film of flowing water. The experiment is fairly reproducible. The rhomboid pattern seems distinct from classical ripples, as the two can develop independently and can be superimposed on one another. The rhomboid pattern eventually reaches a steady state, during which it migrates downstream with a homogeneous wavelength and angle.

[49] Both the surprising regularity of the pattern and its existence in very viscous flows are strong indications that 

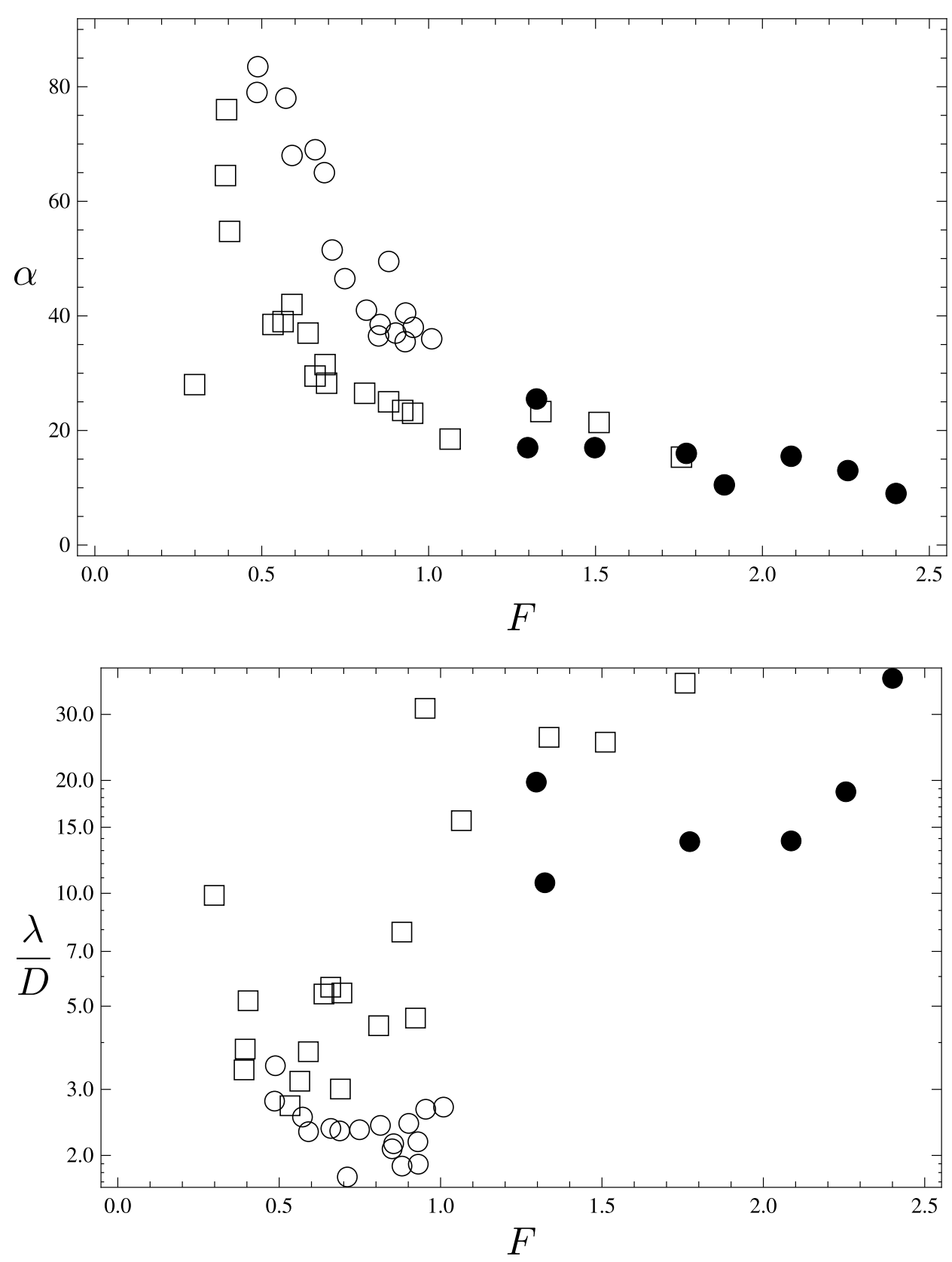

Figure 11. Influence of the Froude number on the rhomboid pattern, at fixed slope. Circles, $S=0.015 \pm$ $10 \%$; squares, $S=0.03 \pm 10 \%$; disks, $S=0.05 \pm 10 \%$.

it is a fundamental morphodynamic instability instead of a subtype or a precursor of classical ripples, although there is no definitive evidence yet in support of this statement. In other words, our experiments tend to indicate that the distinction between rhombus and ripple, well established on morphological grounds alone, is also justified in genesis.

[50] Among the various theories proposed in the literature to explain the formation of rhombus, Woodford's has been the most durable, and many others are variants of it. This theory states that the rhomboid beach pattern can form only in supercritical flows. Our experiments demonstrate that it is not in general the case, even though the claim holds if the fluid viscosity is that of pure water. Since Woodford's

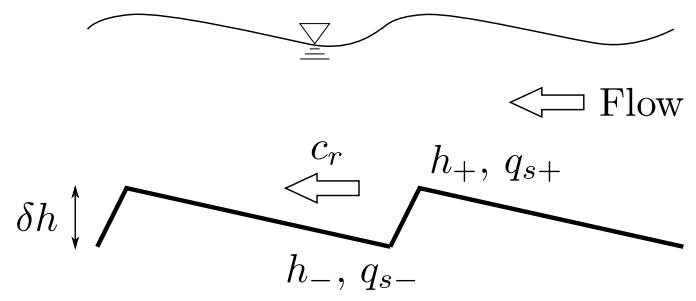

Figure 12. Longitudinal section of the rhomboid pattern. 


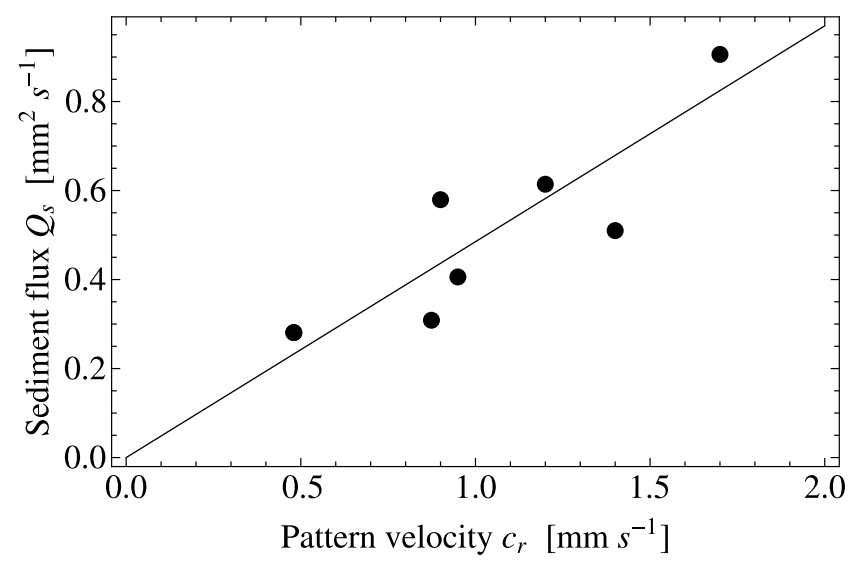

Figure 13. Measured dimensional sediment flux versus measured pattern velocity. The solid line represents the best linear fit, $Q_{s}=c \delta h$, with $\delta h=0.49 \mathrm{~mm}$.

model (as well as any other associated with hydraulic jumps or gravity waves) crucially requires that the Froude number be larger than 1.0, our result indicates that the rhomboid pattern formation is not yet fully understood. Nevertheless, for paleoenvironmental purposes, one may still consider the presence of rhomboid beach pattern as an indicator of supercritical flows, as long as the fluid involved in their formation is pure water.

[51] The dynamics of the experimental rhomboid pattern provides some indications for future theories. Indeed, the typical velocity of this bed form scales with the sediment transport rate, indicating that a complete theory should take sediment transport into account, as opposed to considering the flow only. In other words, our results tend to indicate that the rhomboid pattern is not simply the passive mark of some flow structures, but rather results from the coupling between the flow and the granular bed. If this is confirmed, the rhomboid pattern could become an assessment tool for bed load transport models, a subject of intense present research [Vanoni, 2006; Parker et al., 2003; Charru et al., 2004].

[52] A quantitative model of the rhomboid pattern would also shed light on the role of viscosity in their formation. Are the patterns in subcritical flows distinct from the classical supercritical ones, the viscosity selecting one type or the other? If so, does turbulent bulk viscosity have the same effect on a large-scale rhomboid pattern, as suggested by a referee of the present paper? Those are questions that require both more experiments and a comprehensive theory. Resembling structures formed by laminar and by turbulent flows are not uncommon in geomorphology [Lajeunesse et al., 2010], and their comparison might improve our understanding of both.

[53] The spontaneous emergence of a homogeneous pattern from an initially flat bed, in a reproducible experiment, strikes us as an indication of a linear instability. If this is true, the linear stability analysis of a coupled sediment-flow model should provide an answer to the question of the rhomboid pattern angle. In theory, it could also predicts the pattern wavelength, a characteristic easily measured but predicted by none of the extant theories. We gave this idea an initial trial using the shallow-water equations to model the water flow, but the associated predictions did not match the data any better than Woodford's theory. A three dimensional model is the subject of present research.

\section{Appendix A: Comment on Chang and Simons' Theory}

[54] When Chang and Simons [1970, p. 493] state that "the unsteady terms in the [...] equations can be neglected," they unduly extend a common hypothesis in Geomorphology, namely that the sediment transport time scale is much larger than the dynamical time of the flow. Indeed, this hypothesis allows one to neglect the time derivatives in the flow equations, but on no account can it be used to neglect time in the Exner equation [Parker, 1976]. By doing so, Chang and Simons [1970] reduce their model to a classical hydraulic model that does not take sediment motion into account. Consequently, they could as well remove their equation (8) and not consider the sediment flux $q_{1}$ as a variable.

[55] The above remark implies that Chang and Simons [1970] use the hydraulic model of Woodford [1935], and thus should end up with the same formula for the static wave angle $\alpha$. A mistake in their analysis lead them to propose $\alpha=$ $\arctan (1 / F)$ instead of Woodford's $\alpha=\arcsin (1 / F)$. Indeed, on page 494 , the authors define the determinant

$$
N=\left|\begin{array}{cccccccc}
u & w & 0 & 0 & g & 0 & 0 & 0 \\
0 & 0 & u & w & 0 & g & 0 & 0 \\
h & 0 & 0 & h & u & w & 0 & 0 \\
0 & \frac{w q_{1}}{u^{2}} & 0 & -\frac{q_{1}}{u} & 0 & 0 & -1 & -\frac{w}{u} \\
d x & d z & 0 & 0 & 0 & 0 & 0 & 0 \\
0 & 0 & d x & d z & 0 & 0 & 0 & 0 \\
0 & 0 & 0 & 0 & d x & d z & 0 & 0 \\
0 & 0 & 0 & 0 & 0 & 0 & d x & d z
\end{array}\right|,
$$

where $h, u, w$ and $q_{1}$ are the flow depth, the downstream velocity, the transverse velocity, and the longitudinal sediment flux, respectively. The elementary displacements $d x, d y$ and $d z$ correspond to longitudinal, transverse and vertical directions. Later, the authors present equation (13), namely

$$
(w d x-u d z)^{2}\left((w d x-u d z)^{2}-g h(d x)^{2}\right)=0,
$$

as a condition for $N$ to vanish. The correct equation is actually $(w d x-u d z)^{2}\left(d z^{2}\left(g h-u^{2}\right)+2 d x d z u w+d x^{2}\left(g h-w^{2}\right)\right)=0$.

In turns, this imposes

$$
\frac{d x}{d z}= \pm \sqrt{\frac{u^{2}}{g h}-1}
$$

instead of their equation (15), for a vanishing transverse velocity $W$. Given that $d x / d z=1 / \tan (\alpha)$, one recovers Woodford's formula after some arithmetic.

[56] Acknowledgments. It is our pleasure to thank Clément Narteau, Stéphane Zaleski, François Charru, Philip Hall, Alexander Petroff, and Philippe Claudin for fruitful discussions. We also wish to express appreciation to Brandon McElroy and Paul Carling for their helpful suggestions about the bibliography, as well as to Antonio Vieira and Yves Gamblin for the building of the experimental setup. 


\section{References}

Allen, J. R. L. (1982), Developments in Sedimentology, vol. 30B, Sedimentary Structures - Their Character and Physical Basis, vol. 1, Elsevier, Amsterdam.

Barenblatt, G. I. (1996), Scaling, Self-Similarity, and Intermediate Asymptotics, 386 pp., Cambridge Univ. Press, Cambridge, U. K.

Best, J. (1992), On the entrainment of sediment and initiation of bed defects: Insights from recent developments within turbulent boundary layer research, Sedimentology, 39, 797-811.

Chang, H. Y., and D. B. Simons (1970), The bed configuration of straight sand beds when flow is nearly critical, J. Fluid Mech., 42, 491-495.

Charru, F. (2006), Selection of the ripple length on a granular bed sheared by a liquid flow, Phys. Fluids, 18, 121508, doi:10.1063/1.2397005.

Charru, F., and H. Mouilleron-Arnould (2002), Instability of a bed of particles sheared by a viscous flow, J. Fluid Mech., 452, 303-323, doi:10.1017/S0022112001006747.

Charru, F., H. Mouilleron, and O. Eiff (2004), Erosion and deposition of particles on a bed sheared by a viscous flow, J. Fluid Mech., 519, 55-80, doi:10.1017/s0022112004001028.

Coleman, S. E., and B. Eling (2000), Sand wavelets in laminar openchannel flows, J. Hydraul. Res., 38(5), 331-338.

Collinson, J. D., and D. B. Thompson (1982), Sedimentary Structures, 194 pp., Allen and Unwin, North Sydney, N.S.W., Australia.

Daerr, A., P. Lee, J. Lanuza, and É. Clément (2003), Erosion patterns in a sediment layer, Phys. Rev. E, 67(6), 065201, doi:10.1103/PhysRevE.67. 065201 .

Devauchelle, O., C. Josserand, and S. Zaleski (2007), Forced dewetting on porous media, J. Fluid Mech., 574, 343-364, doi:10.1017 S0022112006004125.

Devauchelle, O., L. Malverti, É. Lajeunesse, F. Métivier, P.-Y. Lagrée, C. Josserand, and S. Zaleski (2008), Erosion shock wave in laminar flumes, in Proceedings of Marine and River Dune Dynamics III: International Workshop, vol. 3, edited by D. Parsons, T. Garlan, and J. Best, pp. 59-62, Univ. of Leeds, Leeds, U. K.

Docoslis, A., R. F. Giese, and C. J. van Oss (2000), Influence of the waterair interface on the apparent surface tension of aqueous solutions of hydrophilic solutes, Colloids Surf. B, 19(2), 147-162.

Gyr, A., and A. Schmid (1989), The different ripple formation mechanism, J. Hydraul. Res., 27(1), 61-74.

Hoyt, J. H., and V. J. Henry (1963), Rhomboid ripple mark: Indicator of current direction and environment, J. Sediment. Res., 33(3), 604-608.

Ikeda, H. (1983), Experiments on bedload transport, bed forms, and sedimentary structures using fine gravel in the 4-meter-wide flume, Pap. 2, pp. 1-78, Environ. Res. Cent., Ibaraki, Japan.

Johnson, D. W. (1919), Shore Processes and Shoreline Development, John Wiley, New York.

Karcz, I., and D. Kersey (1980), Experimental study of free-surface flow instability and bedforms in shallow flows, Sediment. Geol., 27(4), 263-300.

Lajeunesse, E., L. Malverti, P. Lancien, L. Armstrong, F. Métivier, S. Coleman, C. E. Smith, T. Davies, A. Cantelli, and G. Parker (2010),
Fluvial and submarine morphodynamics of laminar and near-laminar flows: A synthesis, Sedimentology, 57, 1-26.

Malverti, L., E. Lajeunesse, and F. Métivier (2008), Small is beautiful: Upscaling from microscale laminar to natural turbulent rivers, J. Geophys. Res., 113, F04004, doi:10.1029/2007JF000974.

McMullen, R. M., and D. J. P. Swift (1967), An occurrence of large-scale rhomboid ripples, Minas Basin, Nova Scotia, J. Sediment. Res., 37(2), 705-706.

Morton, R. A. (1978), Large-scale rhomboid bed forms and sedimentary structures associated with hurricane washover, Sedimentology, 25, 183-204.

Orszag, S. A., and L. C. Kells (1980), Transition to turbulence in plane Poiseuille and plane Couette flow, J. Fluid Mech., 96, 159-205.

Otvos, E. G. (1965), Types of rhomboid beach surface patterns, Am. J. Sci., 263(3), 271-276.

Parker, G. (1976), On the cause and characteristic scales of meandering and braiding in rivers, J. Fluid Mech., 76, 457-480.

Parker, G., G. Seminara, and L. Solari (2003), Bed load at low Shields stress on arbitrarily sloping beds: Alternative entrainment formulation, Water Resour. Res., 39(7), 1183, doi:10.1029/2001WR001253.

Singh, I. B. (1969), Primary sedimentary structures in Precambrian quartzites of Telemark, southern Norway and their environmental significance, Nor. Geol. Tidsskr., 49, 1-31.

Spearman, C. (1904), The proof and measurement of association between two things, Am. J. Psychol., 15(1), 72-101.

Stauffer, M. R., A. Hajnal, and D. J. Gendzwill (1976), Rhomboidal lattice structure: A common feature on sandy beaches, Can. J. Earth Sci., 13(12), 1667-1677.

Straaten, L. (1953), Megaripples in the Dutch Wadden Sea and in the Basin of Arcachon (France), Geol. Mijnbouw, 15, 1-11.

Thompson, W. O. (1949), Lyons sandstone of Colorado Front Range, Am. Assoc. Pet. Geol. Bull., 33(1), 52-72.

Vanoni, V. A. (2006), Manuals and Reports on Engineering Practice, vol. 54, Sedimentation Engineering: Theory, Measurements, Modeling, and Practice, 424 pp., Am. Soc. Civ. Eng., Reston, Va.

Venditti, J. G., M. A. Church, and S. J. Bennett (2005), Bed form initiation from a flat sand bed, J. Geophys. Res., 110, F01009, doi:10.1029/ 2004JF000149.

Williamson, W. C. (1887), On some undescribed tracks of invertebrate animals from the Yoredale rocks, and on some inorganic phenomena, produced on tidal shores, simulating plant remains, Mem. Proc. Manchester Lit. Philos. Soc., 3(10), 19-29.

Woodford, A. O. (1935), Rhomboid ripple mark, Am. J. Sci., 29(174), $518-525$.

O. Devauchelle, É. Lajeunesse, L. Malverti, and F. Métivier, Laboratoire de Dynamique des Fluides Géologiques, Institut de Physique du Globe de Paris, F-75252 Paris, France. (devauche@mit.edu; malverti@ipgp.jussieu.fr) C. Josserand and P.-Y. Lagrée, Institut Jean Le Rond d'Alembert, Université Pierre et Marie Curie, CNRS, F-75252 Paris, France. 